II Bulletin d'analyse phénoménologique XV 2, 2019

ISSN 1782-2041 https://popups.uliege.be/1782-2041/

DOI $10.25518 / 1782-2041.1058$

\title{
Braucht die Logik Objekte? Die Ontologie logischer Gegenstände im Tractatus und Erfahrung und Urteil
}

\author{
Miguel OHNESORGE \\ Universität Kassel, Universidade do Porto
}

\begin{abstract}
Ludwig Wittgenstein's Tractatus logico-philosophicus and Edmund Husserl's Experience and Judgement (Erfahrung und Urteil) are based on remarkably different conceptual frameworks and methodologies. After analyzing their respective accounts on the foundations of (formal) logic, I map out their common aims and different conclusions. I hold that Husserl and Wittgenstein both use the epistemic necessity of the existence of logical relations among things as an argument against philosophical skepticism, but their different epistemological convictions lead them to decisively diverging accounts of the nature of those relations. Wittgenstein assumes a syntactic correspondence theory of truth, which identifies general logical form as necessary condition for accurate representation, apparent in the existence of local truth-functions between propositions. As logical form is the (transcendental) necessary condition of every meaningful proposition, he infers that it is itself not representable and without ontological status. Husserl, by contrast, does not draw from a correspondence theory, but from a processual theory of certainty and truth, which offers genetic instead of categorical distinctions between perception and the logical relations apparent in conceptual knowledge. His theory of formal logic ultimately offers a coherent ontology for logical objects, which avoids logical mysticism.
\end{abstract}

Keywords Logic, ontology, Husserl, Wittgenstein, Tractatus, foundations of logic. 


\section{Einleitung}

Die Trennung der „,westlichen“ Philosophie in „,analytic“ und „continental“ scheint trotz zunehmender Kritik tagesaktuell und identitätsstiftend zu bleiben. Im Ende 2013 erstveröffentlichten Bloomsbury Companion to Analytic Philosophy spricht Howard Robinson von einem postkantischen kontinentalen „rhetorical solipsism“ zwischen monolithischen Denksystemen. ${ }^{1}$ Ohne eine Positionierung hinsichtlich der zugrundeliegenden, mittlerweile intellektuell recht verstaubten Debatten zu beziehen, lässt sich dieser zentrale Anklagepunkt solcher Streitschriften, nämlich der Vorwurf von angeblich hermetisch isolierten Einzelwerken, im Hinblick auf die von Edmund Husserl begründete phänomenologische Philosophie recht einfach falsifizieren. Oft als beinahe idealtypisch kontinentalphilosophisch verstanden, hat sie seit Husserls grundlegenden Arbeiten nicht bloß konstitutive Referenzbegriffe, sondern steht in einem lebendigen und teils fundierenden Austauschverhältnis mit einem weiten Spektrum philosophischer wie auch soziologischer Traditionen. ${ }^{2}$ Nicht zuletzt haben phänomenologische Grundbegriffe auch vermehrt eine breitere Rezeption in ,analytischen“ Publikationen gefunden. Vor allem die unter dem Etikett Philosophy of Mind gebündelten Diskurse, sind nicht nur längst kommensurabel mit phänomenologischer Theorie gemacht worden, sondern beherbergen vermehrt phänomenologische Stellungnahmen. ${ }^{3}$

Die gegenwärtige Phänomenologie ist daher also nicht bloß alles andere als monolithisch, sondern ihr lebendiges Austauschverhältnis mit traditionell als ,analytisch“ verstandenen Theorien hat sich als äußerst fruchtbar erwiesen. Eines der Schlüsselwerke der analytischen Tradition, Ludwig Wittgensteins

\footnotetext{
${ }^{1}$ Howard Robinson, "Coda B: Analytic versus Continental," In The Bloomsbury Companion to Analytic Philosophy, hrsg. v. Ders. u. Barry Dainton (London: Bloomsbury, 2013), 576.

2 Dan Zahavi, Husserl's Legacy: Phenomenology, Metaphysics, and Transcendental Philosophy (Oxford: Oxford UP, 2017), 1.

${ }^{3}$ Vgl. bspw. Dan Zahavi, "Internalism, externalism, and transcendental idealism," Synthese 160 (2008), 355-374; John J. Drummond, Intentionality without Representationalism, In The Oxford Handbook of Contemporary Phenomenology, hrsg. v. Dan Zahavi (Oxford: Oxford UP, 2012), 115-133 (als jüngste Positionierung in der sogenannten East-Coast - West-Coast Debatte, um Husserls Noemabegriff); David Woodruff Smith u. Amie L. Thomasson, Hrsg., Phenomenology and Philosophy of Mind (Oxford, Oxford UP, 2005); Maxime Doyon, "Philosophy of Mind," In Husserl Handbuch: Leben, Werk, Wirkung, hrsg. v. Sebastian Luft u. Maren Wehrle (Stuttgart: J.B. Metzler, 2017), 320-325.
} 
Frühwerk Tractatus logico-philosophicus (dt. Originaltitel: Logischphilosophische Abhandlung), bildet dabei allerdings eine Leerstelle.

Seine teils diametral auseinanderklaffenden Interpretationslinien ${ }^{1}$ trugen hierzu mit großer Sicherheit genauso bei wie der nicht unbegründete Eindruck einer theoretischen Inkommensurabilität mit Analysen logischen Denkens seitens der phänomenologischen Philosophie. Wie Husserl bereits 1900 im Vorwort der Logischen Untersuchungen anmerkte, bezieht die Phänomenologie ihre Legitimität grade aus dem Umstand, dass sie die „reine Logik", ebenso wie das klassische Terrain der Erkenntnistheorie, stets als etwas tiefergehend zu begründendes auffasst. Diese meta-logische und metaepistemologische Methode steht im scharfen Kontrast zum Programm des Tractatus, dessen „Grundgedanken“ es darstellt, dass sich „die [transzendentale] Logik der Tatsachen nicht vertreten lässt" — weder durch die Phänomenologie, noch durch irgendeine Notation oder Theorie überhaupt. ${ }^{2}$

Bei allen Unterschieden lässt sich trotzdem bereits vorläufig ein klar abgestecktes gemeinsames Themenfeld zwischen Wittgensteins Frühphilosophie und wesentlichen Anliegen von Husserls phänomenologischen Analysen anstellen, an welches eine vergleichende Untersuchung anknüpfen kann. Sowohl Husserl als auch Wittgenstein geht es darum, eine Darstellung über Form und Grundlagen logischer Sätze vorzulegen, auch wenn letzterer diesbezüglich vornehmlich negativ argumentiert. Auf Basis jener Gemeinsamkeit verfolgt diese Arbeit ein komparatives und ein kritisches Anliegen. So wird erstens die transzendentale Konzeption der Logik im Tractatus mit Husserls genetisch-phänomenologischer Theorie der Logik verglichen, welche dieser in seinem Spätwerk Erfahrung und Urteil entwickelte. Darauf aufbauend wird aber zusätzlich zweitens eine phänomenologische Kritik an der Logikkonzeption des Tractatus formuliert. Hierbei soll jedoch ebenfalls auf

\footnotetext{
${ }^{1}$ James Conant u. Silver Bronzo, „Resolute Readings of the Tractatus,“ in: A Companion to Wittgenstein, hrsg. v. Hans-Johann Glock u. John Hyman (West-Sussex: Wiley-Blackwell, 2017), 175-195. Zentrale Debatten drehen sich um Wittgensteins Gegenstandsbegriff, welche teils grundlegend unterschiedliche Gesamtinterpretation hervorbrachten, sowie die starke „Unsinnsinterpretation“, welche entgegen der geläufigen Interpretationen alle Sätze des Buches als Scheinsätze versteht. Eine bezeichnende breit rezipierte Fehlinterpretation ist etwa Jürgen Habermas Diagnose eines „Sprachtranszendentalismus“. Siehe Jürgen Habermas, Zur Logik der Sozialwissenschaften (Frankfurt a.M.: Suhrkamp, 1982), 213. Wie weiter unten ausgeführt, genügt ein oberflächlicher Blick in den Tractatus, um die transzendentale Rolle der Logik (nicht der Sprache) auszumachen.

2 Ludwig Wittgenstein, Tractatus logico-philosophicus, Logisch Philosophische Abhandlung (Frankfurt a.M.: Suhrkamp, 2014), §4.0311.
} 
die Erkenntnispotentiale eingegangen werden, welche Wittgensteins Buch umgekehrt für eine phänomenologische Theorie der Logik bereithält.

Das Kommensurabilitätsproblem der Theorien bleibt jedoch augenscheinlich, weshalb sich ihm auch hier nicht entzogen werden kann. Dieses reicht weit über stilistische und begriffliche Differenzen hinaus. Wo Wittgenstein eine zurechtgestutzte korrelationalistische Erkenntnistheorie sowie eine auf den Prinzipien der Operation und Funktion basierende Idealnotation logischer Sätze formuliert, verläuft die Argumentationslinie von Erfahrung und Urteil über eine phänomenologische Analyse der Grundlagen

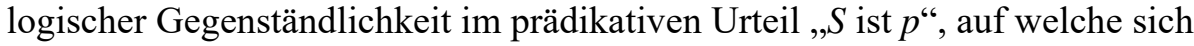
alle anderen prädikativen Urteilsformen zurückführen ließen. Dessen phänomenologische Substrat- und Modalitätsgrundlagen werden in der vorprädikativen Erfahrung und dem prädikativen Denken analysiert und damit im Rahmen einer phänomenologischen Transzendentalphilosophie fundiert. Genauer gesagt, der Tractatus denkt Logik, oder logische Form, als allgemeinen Ausgangs- und Endpunkt jeder Theorie, während in Erfahrung und Urteil logische Gegenständlichkeiten als besondere Gegenstandstypen betrachtet werden, welche ausgehend von ihrer Verschiedenheit und Abhängigkeit von weiteren Gegenstandstypen, sowie einer erfahrenden, erkennenden und urteilenden Subjektivität in einer umfassenderen Theorie analysiert werden.

Um den jeweiligen begrifflichen und argumentativen Feinheiten gerecht zu werden, werden erst beide Ansätze einzeln in Kapitel 2 und 3 in ihren Grundzügen dargestellt. Die Argumentation in Wittgensteins Tractatus wird hier unter dem Gesichtspunkt seiner transzendentalen Konzeption der Logik und der logischen Form, sowie deren Herleitung wiedergegeben. Die Darstellung von Husserls Analyse konzentriert sich wiederum auf die Grundlagen des Urteilens und logischer Gegenständlichkeit in dem prädikativen Denken und vor allem der vorprädikativen Erfahrung, da sich hier am offensichtlichsten die komparativen Besonderheiten seiner phänomenologischen Theorie der Logik zeigen. Notwendigerweise fallen hiermit in beiden Fällen interessante Theoreme aus dem analytischen Raster, welche ebenfalls im weiten Themenfeld der Philosophie der Logik verortbar sind. ${ }^{1}$ Auf diese argumentativen Skizzen aufbauend werden in Kapitel 4 im Rahmen einer kritischen Gegenüberstellung die zentralen Differenzen der beiden Positionen und ihre Implikationen für eine Philosophie der Logik konklusionsartig gegenübergestellt.

${ }^{1}$ So etwa Wittgensteins Herleitung der Zahl als rein logische Operation und weite Teile von Husserls Theorie des Allgemein-Urteilens. 
Diese Arbeit möchte darlegen, dass Wittgensteins Logiktranszendentalismus ein theoretisch nicht haltbares Unterfangen darstellt und dabei durch seine internen Probleme sogar auf die Notwendigkeit einer genetischontologischen Analyse logischer Gegenstände verweist. ${ }^{1}$ Es soll gezeigt werden, dass Logik nicht transzendental und außerweltlich „,vor aller Erfahrung" verstanden werden kann, ${ }^{2}$ sondern nur eingebettet in das alltägliche subjektive Erfahren und Urteilen. Husserl liegt richtig damit, Philosophie nicht bei der Logik enden zu lassen und Logik nicht von ihrer lebendigen Einbettung in die Welt abzusondern.

\section{Logik im Tractatus}

\subsection{Programmatik und Ausganspunkt}

Der Tractatus und seine Rezeption sind gezeichnet von einem beinahe ironischen Nebeneinander von Komplexität und Einfachheit. Zum einen glaubte Wittgenstein, dass die letztendliche Bedeutung der ,ausgedrückten Gedanken" möglicherweise auf Dauer unverstanden bliebe und sah sich konstant missverstanden. ${ }^{3}$ Zum anderen liefert bereits das Vorwort eine scheinbar simple Zusammenfassung des ,ganzes Sinnes des Buches“: „Was sich überhaupt sagen lässt, lässt sich klar sagen; und wovon man nicht reden kann, darüber muss man schweigen.“4 Was explizit bedeute, dass „dem Ausdruck der Gedanken“" (nicht dem Denken selbst) eine Grenze gezogen werde, womit alle philosophischen „Probleme“ beseitigt wären. ${ }^{5}$

Um diesen „Sinn des Buches“ aber wirklich erschließen zu können, muss es im diskursiven Kontext zu den Arbeiten zu Logik und Notation von

\footnotetext{
${ }^{1}$ Dan Zahavi, Husserls Phänomenologie (Tübingen: Mohr Siebeck, 2009), 117. Wie Dan Zahavi treffend anmerkt, ist Husserls Begriff einer genetischen Phänomenologie sehr klar gefasst. Es geht nicht darum da Genesis von $\mathrm{x}$, d.h. dessen prozessualkonstituierende Veränderung selbst zu protokollieren, sondern darum die Wesensstrukturen der Konstitution zu analysieren, ohne den selbst wesenhaften Charakter der „Sedimentation“ von Bewusstseinsinhalten auszublenden. Dan Zahavi, Husserls Phänomenologie (Tübingen: Mohr Siebeck, 2009), 117.

${ }^{2}$ TLP, §5.552.

${ }^{3}$ Ebd., 8.

${ }^{4}$ Ebd., 7.

${ }^{5}$ Ebd., 8.
} 
Gottlob Frege und vor allem Bertrand Russell betrachtet werden. ${ }^{1}$ Wittgenstein sieht Russells philosophische Methode als Grundlage zur Beseitigung der beschworenen philosophischen Probleme. Diese sind schließlich Probleme des Ausdrucks der Gedanken und damit der Sprache, was alle Philosophie zur „Sprachkritik“ macht. Russell ist es dabei zu verdanken eine mögliche Differenz zwischen der „logischen Form“ und der grammatischen Form von Sätzen aufgezeigt zu haben, welche durch eine an der "logischen Form“ orientierten Notation erkennbar wird. ${ }^{2}$ Aus dieser Beobachtung der Möglichkeit der sprachlichen Verwirrung erwächst die programmatische Ausrichtung des Tractatus. Weil Gedanken in ihrer begrifflichen Vertretung in der Sprache ihrer eigentlichen logischen Form durch (i) den Gebrauch sinnloser Zeichen und (ii) unklarer Grammatiken verlieren, entstehen philosophische Probleme. ${ }^{3}$ Philosophie ist daher eine „Tätigkeit“" und sie besteht in „der logischen Klärung der Gedanken“, von der Fehlbarkeit des sprachlichen Ausdrucks. ${ }^{4}$

Die Programmatik des Tractatus, das Ziehen einer Grenze für die Sinnhaftigkeit des sprachlichen Ausdrucks von Gedankens, äußert sich methodisch also als ein Klärungsunterfangen der logischen Form sprachlicher Sätze. Die Philosophie gewährleistet es durch die Ausweisung des sinnvoll Sagbaren, „das Undenkbare von innen durch das Denkbare [zu] begrenzen." ${ }^{\text {"5 }}$ Insoweit steht der Ausgangspunkt des Buches noch ganz im Geist des

\footnotetext{
${ }^{1}$ Besonders: Betrand Russell u. Alfred North Whitehead, Principia Mathematica, Bd. 1-3 (Cambridge: Cambridge UP, 1927); Gottlob Frege, Begriffschrift (Halle: Louis Nebert, 1879).

2 Ebd., §4.0031, Bertrand Russell, „On Denoting,” Mind 14,56 (1905), 482; Michael Kremer, „Russell's Merit,“ In Wittgensteins Early Philosophy (Oxford: Oxford UP 2012), 195-240. Russells paradigmatisches Beispiel stellt die Analyse des Satzes „The present king of France is bald“ dar, dessen alltagssprachliche Negation „The present king of France is not bald" für ihn sowohl falsch als auch wahr sein könnte, weil sie die enthaltene Behauptung der Existenz eines französischen Königs nicht klar aufzeige oder negiere. Eine formale Notation würde dies durch eine Unterscheidung zwischen (i),$\neg \exists \mathrm{x}(\mathrm{KFx} \wedge \forall \mathrm{y}(\mathrm{KFy} \rightarrow \mathrm{y}=\mathrm{x}) \wedge \mathrm{Bx})^{“}$ und (ii) , $\exists \mathrm{x}(\mathrm{KFx} \wedge \forall \mathrm{y}(\mathrm{KFy} \rightarrow \mathrm{y}=\mathrm{x}) \wedge$ $\neg \mathrm{Bx})$ “ verdeutlichen. Das Negationszeichen, „“" wird hierbei dann entweder vor dem Existentialquantor gesetzt, was die Existenz eines $\mathrm{x}$, welches kahler König von Frankreich ist, allgemein negiere (Notation i), oder aber vor „Bx“, was bloß die Kahlheit desjenigen $\mathrm{x}$, welches König von Frankreich ist, negiere (Notation ii); die formallogische Beispielnotation ist eine Übersetzung von Russells Notation aus den Principia Mathematica.

${ }^{3} \mathrm{TLP}, \S 3.325, \S 6.53$.

${ }^{4}$ Ebd., $\$ 4.413$.

${ }^{5}$ Ebd., $\$ 4.114$.
} 
logischen Programms Freges oder Russells. Jedoch besteht sein „Grundgedanke“ darin, dass "sich die Logik der Tatsachen nicht vertreten lässt", beziehungsweise „für sich selber sorgen soll.“2 Es kann also weder eine Aufschlüsselung logischer Form in verschiedene „Typen“ oder eine Verdinglichung der Logik durch selbstreferenzielle Schlussregeln geben. Vielmehr ist es die selbstgesteckte Aufgabe des Tractatus die philosophische Tätigkeit, das heißt die Klärung der Sprache, durch ein neues Verständnis der Logik beziehungsweise der logischen Form zu gewährleisten.

\subsection{Die „Ontologie“ und „Erkenntnistheorie“ der logischen Form}

Wittgensteins programmatische Überlegungen sind keinesfalls voraussetzungslos, sondern basieren auf einer Reihe von scheinbar ontologischen und epistemologischen Unterscheidungen, welche er im ersten Teil des Tractatus expliziert. ${ }^{3}$ Die den Sinn des Buches bestimmenden Leitideen der logischen Form und der durch sie gewährleisten Sinnhaftigkeit müssen deshalb zuerst auf Basis des axiomatischen Begriffsgerüstes ,Satz — Gedanke - Welt' sowie seinen Untergliederungen betrachtet werden.

Die programmatisch angestrebte Sinnhaftigkeit des sprachlichen Ausdrucks besteht in einer optimalen Repräsentation des Wirklichen, also Bestandteilen der Welt („Die gesamte Wirklichkeit ist die Welt.“) ${ }^{4}$ durch den Satz: „Der Satz ist ein Bild der Wirklichkeit. Der Satz ist ein Modell der

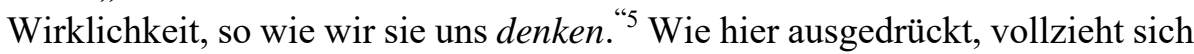

\footnotetext{
${ }^{1}$ Ebd., $\$ 4.032$.

${ }^{2}$ Ebd., §5.473; Die Wichtigkeit des Zusammenbringens des "ganzes Sinnes“" und des „Grundgedankens“ des Buches für die Interpretation tritt am entschiedensten bei Michael Kremers Darstellung hervor. Michael Kremer, „The whole meaning of a book of nonsense: reading Wittgenstein's Tractatus," In The Oxford Handbook of the History of Analytic Philosophy, hrsg. v. Michael Beaney (Oxford: Oxford Up, 2013), 451-487.

${ }^{3}$ Ich schreibe an dieser Stelle „scheinbar“, weil sich der Status von Wittgensteins Korrelationalismus im Laufe der Argumentation im Tractatus noch radikal ändert, wie unter 2.4 dargelegt wird. Eine Darstellung dieser „Erkenntnistheorie“ ist allerdings für das Verständnis des Buches unabdingbar. Das gleiche gilt für den scheinbar fundamentalen Status seiner Gegenstand-Sachverhalt-Tatsache-Welt Ontologie.

4 TLP, $\$ 2.063$

${ }^{5}$ Ebd., $\$ 4.01$.
} 
die dafür notwendige epistemische Brücke zwischen Welt und Satz über den Gedanken, welcher ,das logische Bild der Tatsachen“ ist. ${ }^{1}$

An diesem Punkt wird nun eine genaue Analyse der Architektonik von Wittgensteins Weltbegriff notwendig. Die Welt wird beschrieben als ,alles was der Fall ist" und somit als "Gesamtheit der Tatsachen, nicht der Dinge." ${ }^{\text {"2 }}$ Tatsachen sind ihrerseits alle Modifikationen von ihnen zugrundeliegenden „Sachverhalten“, in denen Gegenstände konfiguriert sind. ${ }^{3}$ Die Feststellung, dass die Welt „,der Fall ist" darf an dieser Stelle noch nicht als Hinweis auf eine durch sprachliche Sätze mit Wahrheitskonditionen konstituierte Welt gedeutet werden. ${ }^{4}$ Vielmehr ist hier „was der Fall ist“ schlicht eine realistische ontologische Setzung, die als Grundbedingung für gedankliche und sprachliche Repräsentation überhaupt fungiert. Diese für die Repräsentation ontologisch notwendige Welt verweist im gleichen Sinne auf die Notwendigkeit ihrer Bestandteile. Ausgehend von der Verknüpfung in komplexen Tatsachen ergibt sich so notwendigerweise die ontologische Grundeinheit des „Gegenstandes“, der wiederum wesenhaft durch das „Vorkommen im Sachverhalt“ 5 bestimmt ist:

Nur wenn es Gegenstände gibt, kann es eine feste Form der Welt geben. [...] Das Ding ist selbständig, insofern es in allen möglichen Sachlagen vorkommen kann, aber diese Form der Selbständigkeit ist eine Form des Zusammenhangs mit dem Sachverhalt, eine Form der Unselbständigkeit. ${ }^{6}$

Die zu repräsentierende Welt ist demnach:

(i) Verknüpft, weshalb es keine Gegenstände außerhalb des Sachverhalts gibt, und letztere auf erstere verweisen - nicht umgekehrt.

(ii) In ihrer komplexen Tatsachenstruktur von den fundamentalen Sachverhalten und darin konfigurierten Gegenständen abhängig.

\footnotetext{
${ }^{1}$ Ebd., $\S 3$.

${ }^{2}$ Ebd., $\$ 1, \S 1.1$.

${ }^{3}$ Ebd., $2.027, \S 2.03, \S 2.032$.

${ }^{4}$ So etwa prominent bei Habermas, Logik der Sozialwissenschaften, 213; oder Markus

Gabriel, Warum es die Welt nicht gibt (Berlin: Ullstein, 2013), 48-50.

${ }^{5}$ Ebd., $\$ 2.011$.

${ }^{6}$ Ebd., $\$ 2.0211, \S 2.026$.
} 
Die „Substanz“ der Welt besteht aber nicht in dieser Struktur ihres Inhalts, sondern in der „Form“ allgemein. ${ }^{1}$ Die allgemeine Form ist erst die „Möglichkeit“ der Struktur, das heißt des konfigurierten Zusammenhängens überhaupt. ${ }^{2}$ Diese allgemeine, grundlegendste Form der Wirklichkeit ist die logische Form und zeigt sich schlicht darin, dass die Welt eine zusammenhängende ist. ${ }^{3}$ Die Strukturen einzelner Tatsachen und Sachverhalte sind besondere Manifestationen dieser allgemeinen logischen Form. Vereinfacht: Alle möglichen Sachlagen (Tatsachen, Sachverhalte) sind logisch, aber die Struktur der einzelnen ,lokalen“ Sachlage kann nie die logische Form insgesamt abbilden. Diese zeigt sich im logischen Raum, der von jeder „Lokalisierung“ bereits vorausgesetzt wird. ${ }^{4}$

Hiervon ausgehend lässt sich nun auch die korrelationalistische Epistemologie des Gedankens und Satzes erläutern. „Wir [die Erkenntnissubjekte] machen uns Bilder der Tatsachen."5 Die Bedingung der Möglichkeit der bildhaften Repräsentation der Welt besteht in der Isomorphie zwischen der Epistemischen und Repräsentierten durch die allgemeine logische Form. Subjekt und Welt sind gleichermaßen logisch formatiert, denn schließlich ist auch „das Bild [...] eine Tatsache“ in derselben logischen Welt. ${ }^{6}$ Der Gedanke, die grundlegende epistemische Einheit, bildet notwendigerweise direkt logisch ab. ${ }^{7}$ Er ist damit „,der sinnvolle Satz“ ${ }^{8}$ Somit ist auch der programmatisch bestimmende Sinnbegriff des Tractatus klar. Der Sinn ist demnach das, „was das Bild darstellt. ${ }^{\text {" } 9}$ Denken ist dabei keine ontologische Sphäre zwischen Satz

\footnotetext{
${ }^{1}$ TLP, $\S 2.024, \S 2.025$.

${ }^{2}$ Ebd., $\$ 2.033$.

3 Ebd., \$2.18. Dies steht in keinerlei Widerspruch zur späteren Definition des Wahrheitskonditionals von Elementarsätzen als notwendigerweise unabhängig von anderen Elementarsätzen. Denn sobald die durch Elementarsätze beschriebenen Sachverhalte in komplexen Situationen zu Tatsachen verknüpft werden (Die Farbe Rot tritt nicht als reines metaphysisches Rot auf, sondern als rot einer spezifischen Reklame oder Farbpalette) zeigt sich offensichtlich, dass der unabhängige Wahrheitswert den Sachverhalt nicht aus den Verknüpfungen der Tatsachen-Welt löst. Siehe Abschnitt 2.3.

${ }^{4}$ Ebd., $\$ 3.42$.

${ }^{5}$ Ebd., $\$ 2.1$.

${ }^{6}$ Ebd., $\$ 2.141$.

${ }^{7}$ Ebd., $\$ 3$.

${ }^{8}$ Ebd., $\$ 4$.

${ }^{9}$ Ebd., $\$ 2.221$.
} 
und Welt, sondern gewissermaßen die Erscheinungsweise der Welt. ${ }^{1}$ Die gedankliche Repräsentation ist daher immer die direkte Repräsentation der Welt und somit notwendigerweise sinnvoll. Dies erklärt wiederum, warum der sinnvolle Satz ein „Bild der Wirklichkeit ist, wie wir sie uns denken.“2

Wie der Gegenstand nur im Sachverhalt auftritt, ist das gedankliche Bild ein zusammengesetztes und das Zeichen hat nur im Bild des Satzes Sinn. Repräsentation muss die allgemeine Formatierung der logischen Form und die spezifische Formatierung einer besonderen Sachlage besitzen, um repräsentieren zu können. ${ }^{3}$ Durch das Bild ist die Grundlage der Wahrheit und Falschheit gegeben. Seine formale Kommensurabilität mit der Wirklichkeit ist die Möglichkeitsbedingung von Wahrheit und Falschheit, welche sich durch vergleichende Betrachtung offenbaren.

Es ist an diesem Punkt notwendig die herausgearbeitete Differenz zwischen Gedanke und Satz schärfer zu erläutern. Beide sind eingebettet in die logische Form und beide repräsentieren diese in spezifischen Sachlagen bildhaft. Trotzdem ist der Gedanke immer sinnhaft, der sprachliche Satz hingegen nicht. Diese Differenz gründet sich in der Fehlbarkeit der Sprache. Dadurch, dass Begriffe mehrfach belegt werden und Grammatik die der Alltagssprache zugrundeliegende logische Form von Sätzen verschleiern kann, ist es möglich Sätze mit sinnlosen, das heißt nicht repräsentierenden Zeichen zu formulieren. ${ }^{4}$ Somit ist Sprache zwar strenggenommen immer noch notwendigerweise logisch, aber potentiell sinnlos:

Jeder mögliche Satz ist rechtmäßig gebildet, und wenn er keinen Sinn hat, so kann das nur daran liegen, dass wir einigen seiner Bestandteile keine Bedeutung gegeben haben. (Wenn wir auch glauben, es getan zu haben.) So sagt »Sokrates ist identisch « darum nichts, weil wir dem Wort »identisch« als Eigenschaftswort keine Bedeutung gegeben haben. Denn, wenn es als Gleichheitszeichen auftritt, so symbolisiert es auf ganz andere Art und Weise — die bezeichnende Beziehung ist eine andere, — also ist auch das Symbol in

\footnotetext{
${ }^{1}$ Hier ist die unter $\$ 5.6331$ formulierte Analogie des Gesichtsfelds aufschlussreich. Das Gesichtsfeld einer Person zeigt sich uns keinesfalls drittpersonal, sondern immer erstpersonal. Die Welt wird erstpersonal erschlossen, weshalb alleine die Existenz sinnloser Gedanken in Wittgensteins korrelationalistischer Theorie das Fundament jeder Ontologie erodieren würden.

${ }^{2}$ Ebd., $\S 4.01$.

${ }^{3}$ TLP, $\S 3.3, \S 3.31, \S 3314$.

${ }^{4}$ „Sinnlos“ wird im Folgenden im Sinne des Tractatus gebraucht.
} 
beiden Fällen ganz verschieden; die beiden Symbole haben nur das Zeichen zufällig miteinander gemein. ${ }^{1}$

So lässt sich nun auch die Falschheit eines Bildes grundlegend von der Sinnlosigkeit eines Satzes unterscheiden. Auch ein falsches Bild bildet logisch ab und seine Falsifizierung sagt etwas über die Welt aus. Ein sinnloser Satz hingegen besitzt keinen Weltbezug und damit auch keinen Wahrheitswert, weil er aufgrund eines oder mehrerer nicht abbildender Zeichen überhaupt nicht verifiziert oder falsifiziert werden kann.

Durch diese scheinbar ontologischen und epistemologischen Theoreme, lässt sich die Intention hinter der programmatischen Freilegung der logischen Form der Sprache spezifizieren. Diese besteht folglich darin, dass sich durch eine von Unklarheiten befreite Notation zeigt, wann Zeichen sinnlos sind und wann nicht.

\subsection{Die Notation logischer Form}

Wittgenstein entwickelt im weiteren Verlauf des Buches eine Notation, welche die philosophische Klärungsaufgabe bewältigen soll. Diese bedient sich „formaler Begriffe,“ welche durch Variablen ausgedrückt werden und sprachlichen Ausdrücke auf ihre allgemeinen Formen zurückführen sollen. Das Herzstück dieser Notation bilden die formalen Begriffe der Operation und Funktion. Die Operation $\left(\Omega^{\circ}\right)$ stellt die Übersetzung eines Satzes in einen anderen dar, das heißt eine beliebig formalisierte Modifikation. Die Funktion hingegen stellt wie bei Frege und Russell die logische Beziehung zwischen Argumenten dar. ${ }^{2}$ Durch bestimmte Operationen treten damit die Funktionen, also das Verhältnis verschiedener Ausdrücke zu Tage.

Es muss nun scharf zwischen den rein logischen und den sinnvollen Sätzen unterschieden werden. Die grundlegendste sinnvolle sprachliche Einheit ist der Elementarsatz. Dieser „,behauptet das Bestehen eines Sachverhaltes" und wird von Wittgenstein durch eine der Variabeln $p, q, r$ notiert. $^{3}$ Ein Elementarsatz ist eine Verkettung von Namen, welche die sprachlichen

\footnotetext{
${ }^{1}$ Ebd., $\$ 54733$.

2 TLP, §3.381, §5.02, §5.3; Das Argument ist der in der Funktion auftretende Ausdruck, also das logisch verknüpfte Zeichen. Argument somit ein noch allgemeinerer formaler Begriff für alles wie auch immer sinnvoll, d.h. im logischen Raum bezeichnete.

${ }^{3}$ Ebd., $\$ 4.21$.
} 
Vertreter der Gegenstände sind. ${ }^{1}$ Folglich zeigt sich der Sinn eines Namen im Elementarsatz, nicht umgekehrt. Elementarsätze sind damit die grundlegenden sinnvollen Beschreibungen der Welt. Würde die Alltagssprache auf die sie zusammenhängenden Elementarsätze zurückgeführt werden, gäbe es eine vollständige grundlegende Beschreibung der Welt und es ließen sich alle anderen sinnvollen Sätze situativ aus diesen bilden. ${ }^{2}$

Im Gegensatz dazu stehen die Sätze der reinen Logik, welche nichts in der Welt bezeichnen. ${ }^{3}$ Dies zeigt sich am anschaulichsten an den Extremfällen der Tautologien und Kontradiktionen, welche aufgestellt werden, weil sie nichts in der Welt bezeichnen können. Sie weisen so darauf hin, wie sich die formalen, also die logischen Eigenschaften sinnvoller Sätze zueinander verhalten. ${ }^{4}$ Trotzdem haben solche sinnlosen Scheinsätze eine Funktion, sie zeigen die formalen Eigenschaften der Welt auf:

Dass die Sätze der Logik Tautologien sind, das zeigt die formalen — logischen - Eigenschaften der Sprache, der Welt. Dass ihre Bestandteile so verknüpft eine Tautologie ergeben, das charakterisiert die Logik ihrer Bestandteile. Damit Sätze, auf bestimmte Art und Weise verknüpft, eine Tautologie ergeben, müssen sie bestimmte Eigenschaften der Struktur haben. Dass sie so verbunden eine Tautologie ergeben, zeigt also, dass sie diese Eigenschaften der Struktur besitzen. ${ }^{5}$

Scheinsätze der Logik sind also Operationen, die verdeutlichen können, was das Bestehen (d.h. der Wahrheitswert) sinnvoller Sätze für das Bestehen anderer mögliche Sätze bedeutet (d.h. deren Wahrheitsfunktion offenlegen). Sie sind damit Funktionen des Wahrheitswertes von bereits aufgestellten

\footnotetext{
${ }^{1}$ Ebd., $\$ 4.22$; Tempsky, 1992), 419-428.

${ }^{3}$ Ebd., §6.1221, §6.124, §6.126.

4 TLP, $\$ 4.461$

${ }^{5}$ Ebd., $\$ 6.124$.
}

${ }^{2}$ Ebd., §4.51, §4.52. Über den Charakter Elementarsätze gab es in der analytischen Philosophie prominente Debatten. Die Namen wurden oft durch nur selbst- und wahrnehmungsreferenzielle Ausdrücke am Beispiel der Farbnamen erklärt, so unter anderem von Wittgenstein selbst: Siehe Friedrich Weismann, Brian F. McGuiness, (Hrsg.), Ludwig Wittgenstein und der Wiener Kreis: Gespräche, aufgezeichnet von Friedrich Waismann (Frankfurt a.M.: Suhrkamp, 1984), 73-74. Die Inkohärenz dieser Auffassung wurde prominent kritisiert von A.J. Ayer: Siehe A.J. Ayer, „The elementary Propositions of the Tractatus", in Sprache und Ontologie: Akten des 6. Internationalen Wittgenstein Symposiums 23. bis 30. August 1981, Kirchberg/Wechsel, Österreich, hsrg. v. Elisabeth Leinfellner et. Al. (Wien: Hölder-Pichler- 
sinnvollen Sätzen, weshalb die allgemeine Form der Wahrheitsfunktion auch immer auf die Existenz von zu verifizierenden/falsifizierenden Sätzen und damit auf das Bestehen von sinnvollen Elementarsätze angewiesen ist. ${ }^{1}$

Ist in der Logik erst die Negationsoperation $\mathrm{N}(\xi)$ definiert — und somit Wahrheit/Falschheit als ihr Ergebnis binär formalisiert -, lassen sich mit ihrer Anwendung auf einen unbestimmten sinnvollen Satz auch alle bekannten logischen Konstanten (Negation, Konjunktion, Disjunktion etc.) definieren, welche Symbole für bestimmte Wahrheitsfunktionen zwischen sinnvollen Sätzen sind. ${ }^{2}$ Die Rolle der logischen Symbole bleibt damit auf die philosophische Tätigkeit des Klärens von Sätzen beschränkt. Sinnvolle Sätze zeigen immer ihre Logik an, lokalisieren sich im logischen Raum. Logische Scheinsätze über Wahrheitsfunktionen können diesen zeigenden Gestus bloß vereinfacht reproduzieren. Nur da die eigentlich unmittelbare logische Form von Sätzen durch unklaren Gebrauch der Sprache verdeckt werden kann, wird es notwendig logische Konstanten zu definieren, welche die logische Form von Aussagen deutlicher aufzeigen. „Die Sätze der Logik sind [also] Tautologien."3 Sie können bloß die logisch-formalen Eigenschaften der repräsentierten Sachlagen zeigend veranschaulichen und damit sinnvolle Sätze klären.

\subsection{Logiktranszendentalismus}

Was sind die Folgen aus dem zeigenden Gestus der Sätze der Logik und ihres tautologischen Charakters? Erstens ist es nun offensichtlich, dass alle logischen Konstanten sinnlose Klärungswerkzeuge sind. Es sollte im klärenden logischen Symbolismus also nur ein Urzeichen definiert werden, nämlich die ,allgemeine Satzform, “4 welche bloß auf das Bestehen mindestens eines Elementarsatzes und der wesenhaften Falsifizier- und Verifizierbarkeit aller abbildenden Sätze hinweist. Auf dieses „Urzeichen“ lassen sich alle weiteren logischen Konstanten zurückführen. Zweitens erübrigt sich jede Theorie bestimmter Schlussregeln in der Logik, da diese bloß das immer

\footnotetext{
${ }^{1}$ Ebd., $\S 6$.

${ }^{2}$ Ebd., $\$ 5.43$.

${ }^{3}$ Ebd., $\$ 6.1$.

${ }^{4}$ Ebd., §6, Diese entspricht der allgemeinen Wahrheitsfunktion,,[p, $\left.\xi, N(\xi)\right]^{“}$ und drückt die wahrheitsfunktionale Relation zwischen einem beliebigen Satz p und einen sinnvollen Elementarsatz $\xi$ aus. Wittgenstein deutet in der Originalnotation durch Striche über den Variablen an, dass diese alle vertretbaren Inhalte auch vertreten. Dies ist an dieser Stelle aber für die die Argumentation unwesentlich.
} 
vorauszusetzende Verständnis der potentiellen Wahr/Falschheit von sinnvollen Sätzen wiedergeben. Sie tragen nicht sonderlich zum Klärungsprozess bei und sind daher definitorischer Ballast. Schlimmer noch suggerieren sie, dass sich die Logik von außen quasi metalogisch reglementieren lasse. ${ }^{1}$

Diese Feststellungen verweisen allerdings keinesfalls auf eine Marginalisierung der Logik. Der sinnlose Charakter der Logik ändert nichts daran, dass das worauf sie hinweist, worauf also auch die Struktur jedes sinnvollen Satzes hinweist, den sinnvollen Ausdruck seinerseits erst möglich macht. Denn ohne die Möglichkeit von Wahrheit und Falschheit von Sätzen könnte es keine sinnvolle Repräsentation geben. Nur da erkennbar ist, dass ein Satz keine Kontradiktion oder Tautologie ist, ist er als sinnvoll erschließbar. Dass die Logik nun bestenfalls eine zeigende Klärung ist, nimmt ihrem „Objekt", der logischen Form, nicht seine Relevanz. Es zeigt lediglich, dass die Sätze der Logik Sätze bleiben und deswegen logische Form ihrerseits auch nur durch ihre Struktur zeigen können. Denn jede Aussage über logische Form wird zu einer Aussage mit und auf Basis logischer Form, wodurch sich deren Form selbst der Bezeichnung entzieht:

Das Bild kann sich aber nicht außerhalb seiner Form der Darstellung stellen. [...] Um die logische Form darstellen zu können, müssten wir uns mit dem Satze außerhalb der Logik aufstellen können, das heißt außerhalb der Welt. ${ }^{2}$

Es zeigt sich im Satz und klarer im logischen Satz nichts anderes als „die logische Form der Wirklichkeit. ${ }^{\text {"3 }}$ Diese grundlegende Bedingung von Sinn, Wahrheit und Falschheit, ist damit nicht weniger als die Bedingung der Möglichkeit von Erkenntnis überhaupt. Die nicht sprachlich zu bezeichnende Logik ,ist transcendental. [sic]“4 Die Verknüpfung in einem logischen Bild macht die Bezeichnung seiner Bestandteile schließlich erst möglich. Der Gegenstand existiert nur im logischen Sachverhalt und das Zeichen hat nur im logischen Satz Sinn.

Dies hat schließlich aber grundlegende Folgen für die Sätze des Tractatus selbst, welche sich als ,unsinnig“ erweisen. Alle Sätze über logische Form sind selbst sinnlose, zeigende Hinweise. Die eigentliche „logische Form der Welt" ist kein semantisches Korrelat von Zeichen, sondern zeigt sich in

\footnotetext{
${ }^{1}$ Anstatt Schlussregeln schlägt Wittgenstein die anschaulicheren Wahrheitstabellen vor. Diese sollen dem Anschein vorbeugen, es würde gegenstandsartige logische Konstanten geben.

${ }^{2}$ TLP, $\$ 2.174, \S 4.12$

${ }^{3}$ Ebd., $\$ 4.121$.

${ }^{4}$ Ebd., $\$ 6.13$.
} 
der durch den Tractatus ermöglichten Perspektive auf die Welt als eine transzendental logisch formatierte. Die oben skizzierte Ontologie und Erkenntnistheorie erweisen sich als sinnloses Hilfsgerüst, als „Leiter“, welche die Leserin nach dem Besteigen ,wegwerfen“ kann. ${ }^{1}$ Denn alle Feststellungen über die Struktureinheiten von Welt und Welterkenntnis sind ihrerseits in der nicht bezeichnend thematisierbaren und transzendentalen logischen Form begründet. Schließlich kann es keine außerlogische „Begründung“ der Logik geben: „Die Logik muss für sich selbst sorgen.“2

So kann Wittgenstein trotz dem fallibelen Charakter der Sprache, der erst die klärende Programmatik des Buches begründet, behaupten „die Grenzen meiner Sprache bedeuten die Grenzen meiner Welt. “3 Die Welt erschließt sich erst durch die Bildung besserer logischer Bilder durch klarere sprachliche Ausdrücke. So wird die der Fall seiende Welt, von einer zu anfangs bloßen Setzung zu einer „Aufgabe,“ welche sich in den empirischen Wissenschaften auf Basis der transzendentalen Logik vollzieht. ${ }^{4}$ Die ,vor aller Erfahrung" stehende Logik ,zeigt" uns die Notwendigkeit der erst begründungslos vorausgesetzten Gegenstände, Sachverhalte und Tatsachen, sobald sich ihre Formatierung in der zu erschließenden Erfahrungswelt offenbart. ${ }^{5}$

\section{Logik in Erfahrung und Urteil}

\subsection{Programmatik und Ausgangspunkt ${ }^{6}$}

Im Gegensatz zum Tractatus steht Erfahrung und Urteil im Rahmen und am Ende eines über Jahrzehnte zusammengetragenen Theoriegerüstes. Es schließt

\footnotetext{
${ }^{1}$ TLP, $\$ 6.54$.

${ }^{2}$ Ebd., $\$ 5.473$.

${ }^{3}$ Ebd., §5.6.

${ }^{4}$ Ebd., $\$ 6.4321$.

${ }^{5}$ Strenggenommen „steht“ Wittgensteins Logik nicht und „ist“ auch nicht, da sie schlicht prädikativ und damit erst recht ontologisch und metaphysisch unbestimmbar bleibt.

${ }^{6}$ Die ersten vier Paragraphen des Buches sind größtenteils auf die editorische Tätigkeit Ludwig Landgrebes zurückzuführen. Dies wird im Folgenden nicht beachtet, da keine der allgemeinen Programmatik potentiell widersprechenden Stellen zitiert wurden. Siehe Dieter Lohmar, „Zu der Entstehung und den Ausgangsmaterialien von Edmund Husserls Werk Erfahrung und Urteil,“ Husserl Studies 13,1 (1996), 31-71; Jagna Brudzinska, „Erfahrung und Urteil,“ In Husserl Handbuch: Leben, Werk, Wirkung, hrsg. v. Sebastian Luft u. Maren Wehrle (Stuttgart: J.B. Metzler, 2017), 104.
} 
an das Problem an, welches Husserl in den Logischen Untersuchungen erst zur Konzeption der Phänomenologie als eigenständiger Metawissenschaft geführt hatte. Die Aufstellung einer Theorie der Logik, welche weder selbstreferenziell-formal oder psychologistischkonzipiert ist. Dies soll durch eine auf Erfahrung und subjektiven Akten basierende Philosophie, welche dennoch eine streng ideale und apodiktische Theorie der Logik als eines ihrer Kernanliegen vertritt, gewährleistet werden. Wie Jitendranath Mohanty es provokativ zuspitzt, lässt sich Husserl Philosophie als ein Weg zwischen Platonismus und Anti-Platonismus interpretieren. ${ }^{1}$

Der theoretische Ausgangspunkt zur Analyse der Logik in Husserls Spätwerk ist dabei vorläufig am klarsten in Abgrenzung zu diesen beiden radikalen Polen bestimmen.

Zum einen muss eine sich mit bloßen formalen Gesetzen der Logik begnügende Theorie fehlschlagen, weil sie ihren Grundbegriff, den Wahrheitswert, nicht sinnhaft bestimmen kann. Die in den leeren Variablen formaler Sätze ausgedrückte Referenz auf „Gegenstände-worüber“ kann nicht auf einen Wahrheitswert befragt werden, solange kein Evidenzbegriff vorliegt. Solch eine Theorie scheitert daran zu bestimmen, wann die Art der Gegebenheit einer Sachlage (Gegenstandsevidenz) eine Verifikation oder Falsifikation gefällter Urteile zulässt (Urteilsevidenz). Grundlegender ignoriert sie damit auch die im Evidenzbegriff wesenhaft verankerten Metaanalysen der erscheinenden Gegenstände und des Bewusstseins, dem sie erscheinen. ${ }^{2}$

Zum anderen muss eine psychologistische Theorie scheitern, da diese bereits eine im objektiven Status existierende Welt voraussetzt. Sie ignoriert die objektivitätskonstituierende Rolle der Logik und somit die effektiven Resultate logischer Bewusstseinsleistungen. Sie setzt ursprünglicher konstituierende Urteile über Wahrheit, Falschheit und ontologische Vergleichsobjekte voraus, was notwendigerweise auf ein schlichtes Annehmen ihres eigentlichen Untersuchungsgegenstandes, der Logik, verweist.

Husserls phänomenologische Alternativbetrachtung der „logischen Problematik" ist dabei weiterhin nicht ohne eine schemenhafte Einbettung in seine weitere Philosophie erschließbar, welche er selbst mehrfach vornimmt. Erfahrung und Urteil schließt an die Formale und transzendentale Logik an, in welcher der Anfang und „die prinzipiellen Fragen“ einer „phänomenologischen Ursprungserhellung des Logischen“ expliziert wurden. Eine

${ }^{1}$ J.N. Mohanty, Edmund Husserl's Theory of Meaning (Dordrecht: Martinus Nijhoff, 1964), 2.

2 Edmund Husserl, Erfahrung und Urteil: Untersuchungen zur Genealogie der Logik (Hamburg: Felix Meiner, 1972), 7-20. 
Variante solch einer Ursprungserhellung wird in Erfahrung und Urteil „in einem Stück durchgeführt." ${ }^{\text {"1 }}$ Dabei beschränkt sich das Buch jedoch auf eine Ursprungsanalyse der klassischen formalen Logik. Diese programmatische Skizze lässt sich durch eine von Husserl selbst angebotenen zweiseitige Abgrenzung von einer phänomenologischen Untersuchung der Konstitution überhaupt (i) und des Logischen überhaupt (ii) näher bestimmen. ${ }^{2}$

Erstens unterscheidet sich Erfahrung und Urteil von dem Grundanliegen einer konstitutiven Phänomenologie. Diese stellt eine transzendentale Erörterung der Konstitution von Welt und Gegenständen überhaupt in ihrem Rückgang auf die transzendentale Subjektivität dar. Dabei muss der von Husserl selbst betonte Gegensatz zum gängigem kantischen Verständnis der Transzendentalphilosophie hervorgehoben werden, ${ }^{3}$ die Jitendranath Mohanty und Dan Zahavi treffend als prinzipientheoretisch abgrenzen. Kants theoretische Philosophie verortet die Bedingung der Möglichkeit von Erkenntnis gewissermaßen negativ durch die statische Bestimmung von erfahrungsunabhängigen apriorischen Prinzipien. Husserls ,evidenztheoretische“ Herangehensweise hingegen verlangt es, das transzendentale Subjekt in seiner intentionalen Bezogenheit auf Gegebenes zu untersuchen. ${ }^{4}$ Die methodische „transzendentale Reduktion“ ist keinesfalls eine rein negative Abstraktion von aller Erfahrung, sondern unternimmt es das Wesen der transzendentalen Subjektivität im Konstitutionsprozess positiv und genetisch zu beschreiben. Dadurch wird der Konstitutionsprozess im Bewusstsein als immanent selbstzeitigend, horizontal und in die Intersubjektivität, fungierende Leiblichkeit sowie eine sich sedimentierende historische Lebenswelt eingebunden charakterisierbar. ${ }^{5}$ Der Kontext dieser transzendentalen Untersuchung der

\footnotetext{
${ }^{1}$ Ebd., 3.

${ }^{2}$ EU, 48-51.

${ }^{3}$ Edmund Husserl, Erste Philosophie I (Dordrecht: Kluwer, 1996), 230-287.

${ }^{4}$ Zahavi, Husserl's Legacy, 123; J.N. Mohanty, The Possibility of Transcendental Philosophy (Dordrecht: Martinus Nijhoff, 1985), 215.

${ }^{5}$ Edmund Husserl, Die Krisis der europäischen Wissenschaften und die transzendentale Phänomenologie:

Eine Einleitung in die phänomenologische Philosophie (Hamburg: Meiner, 2012), 100-102. Die Interpretation des inneren Zeitbewusstseins als präreflexiv intentional selbstzeitigend und die ihr zugrundeliegende Interpretation von Husserls Zeitlichkeitsanalyse allgemein (präreflexiv selbstzeitigender Bewusstseinsstrom — reflexive Zeitlichkeit von thematisierten Akten und Erfahrungen - objektive Zeit der Gegenstände) basiert auf: Zahavi, Husserls Phänomenologie, 90-95.
}

17 
Weltkonstitution spielt zwar eine Rolle in Erfahrung und Urteil, ${ }^{1}$ geht in seiner Fülle jedoch über die Analysen des Buches hinaus, weshalb er in weiten Teilen „bereits vorausgesetzt“ wird. ${ }^{2}$

Zweitens liegt keine Untersuchung der transzendentalen Funktion der Logik überhaupt vor. Erfahrung und Urteil unternimmt es demnach nicht, umfassend zu bestimmen, „was alles im Aufbau der Welt als Anteil logischer Sinnbildung, logischer Leistung zu verstehen " ist. ${ }^{3}$ Es wird zwar festgestellt, dass logische Bewusstseinsleistungen weit über die klassische formale Logik hinaus von zentraler Relevanz sind. Die weitläufigen Bedeutungen dieser Relevanz werden jedoch nicht umfassender erläutert.

Die Ursprungsanalyse der formalen Logik wird in der propädeutischen Einleitung auf einen, „im Zentrum der formalen Logik“ verorteten Untersuchungsgegenstand zugespitzt: Das zumeist sprachliche prädikative Urteil

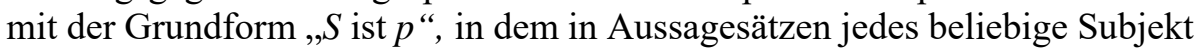
mit beliebigen Prädikaten versehen werden. ${ }^{4}$ Dieses Urteil verweist immer auf ihm zugrundeliegendes Beurteiltes, weshalb die Evidenz eines Urteils auf die Evidenz der Gegebenheit des beurteilten Seienden verweist. Die Urform der Evidenz wird also phänomenologisch als Selbstgegebenheit eines Gegenstandes im Bewusstsein definiert. ${ }^{5}$ Somit verweist die Grundlage der formalen Logik, das evidente Urteilen, auf die ursprüngliche Gegebenheit von beurteilten Gegenständen. Diese finden sich in der Analyse des Bewusstseins nicht nur prädikativ und somit als Substrat prädikativer Urteile, welche bleibenden Erkenntnisbesitz in Sachverhalten schaffen. ${ }^{6}$ Vielmehr sind diese ihrerseits fundiert in bloß erfassenden und bestimmenden, aber nicht reproduzierbaren Urteilen (vorprädikative Urteile). Diese vollziehen sich in der vorprädikativen Erfahrung - beurteilt werden aus einem weltlichen Sinnesfeld affizierende Gegenstände. ${ }^{7}$ Es wird daher ein weiter Urteilsbegriff

${ }^{1}$ EU, S. 49. Husserl nimmt hier diese Kontextualisierung analog zum phänomenologischen Zweischritt von Epoché und Reduktion explizit vor, wenn er vom Rückgang auf die Lebenswelt (Auflösung der naiven Einstellung) und den diese erst konstituierenden subjektiven Leistungen (transzendentale Reduktion) spricht.

${ }^{2}$ EU, 72. Vorausgesetzt werden konkret die kinästhetische (leibliche) Wahrnehmungsdimension des fungierenden Betrachters, die Konstitution von Sinnfeldern, die Konstitution der Zeitlichkeit der Dinge und die reflexive Konstitution präreflexiv erlebter intentionalen Akte als Akte.

${ }^{3} \mathrm{EU}, 50$.

${ }^{4}$ Ebd., 1-5.

${ }^{5}$ Ebd., 11-12.

${ }^{6}$ Ebd., 231-233.

${ }^{7}$ Ebd., 60-66. 
entwickelt, der alle subjektiv vollzogenen Gegenstandskonstitutionen umfasst, also „die Gesamtheit der objektivierenden Ichakte. “" Um diese genetischen Analyse des Urteils in der unmittelbaren Erfahrung streng durchzuführen, stellen Urteile über Individuelles in der äußeren Wahrnehmung die Untersuchungsobjekte der Einzelanalysen dar, da diese die „Strukturen der Welt aufdecken“ und ,jedem praktischen Verhalten zugrunde liegen.“2

Lediglich methodisch ist jedoch die Beschränkung auf deinem einzelnen Subjekt gegebene Gegenständen, da Husserl an anderer Stelle bereits die weitaus grundlegendere Rolle der Intersubjektivität in der transzendentalen Phänomenologie herausgearbeitet hat. ${ }^{3}$

\subsection{Die vorprädikative Erfahrung als Ursprungsort logischer Bewusstseins-} leistung

\subsubsection{Allgemeine Rezeptivität und Modalisierung}

Die vorprädikative Erfahrung erweist sich nicht nur als ursprüngliche gegenständliche Fundierungsdomäne des evidenten Urteils. In ihr finden sich außerdem bereits die intentionalen Modifikationen, welche den Urteilsmodalitäten der Logik zugrunde liegen. In ihrer ausführlichen Betrachtung und Rolle liegt, wie Dieter Lohmar es komparativ umfassend dargelegt hat, das charakteristischste Merkmal von Husserls genetisch-phänomenologischer Theorie der Logik, welche in Erfahrung und Urteil kulminiert. ${ }^{4}$

Die vorprädikative Erfahrung kann näher bestimmt werden als Sphäre der Rezeptivität. Dies steht nur auf den ersten Blick im Widerspruch zur ihrer Relevanz zur Rückverfolgung des weiten Urteilsbegriffs, welcher sie folglich als eine Domäne ichlicher Aktivität ausweist. Vielmehr wird grade aufgezeigt, warum bereits in der Rezeptivität die „niederste Stufe ichlicher Aktivität“

\footnotetext{
${ }^{1}$ Ebd., 63.

${ }^{2}$ Ebd., 67. Zum individuellen Gegenstand als einzig schlicht gegebenen: Ebd., 18-20.

${ }^{3}$ Edmund Husserl, ,Kann es getrennte Subjekte bezogen auf getrennte Welten geben? Bedingungen der Möglichkeit der Koexistenz von Subjekten. Deduktion, dass es nur eine Welt, nur eine Zeit, einen Raum geben kann,“ Ders., Texte zur Phänomenologie der Intersubjektivität II (Den Haag: Nijhoff, 1973), 91-104.

${ }^{4}$ Dieter Lohmar, Erfahrung und kategoriales Denken: Hume, Kant und Husserl über vorprädikative Erfahrung und prädikative Erkenntnis (Dordrecht: Springer, 1999), 274; Ders., „Warum braucht die Logik einer Theorie der Erfahrung?,“ In Phenomenology on Kant, German Idealism, Hermeneutics and Logic: Philisophical Essays in Honor of Thomas M. Seebohm (Dordrecht: Springer, 2000), 149-170.
} 
vorzufinden ist und die Rezeptivität aus phänomenologischer Perspektive nicht alltagssemantisch als ,passiv“ beschrieben werden kann. ${ }^{1}$

Die Rezeptivität fußt auf der „,puren Passivität“, in welcher im Bewusstsein noch nicht spezifisch konstituierte Gegenstände aus auf einem Sinnesfeld heraus affizieren, beispielsweise dem Feld optischer Gegebenheiten, welches „assoziativ“ strukturiert ist. ${ }^{2}$ Der deskriptive Begriff der ,assoziativen Struktur" beschreibt eine „wesensmäßige Form der Gesetzmäßigkeit innerer Genesis" und damit die allgemeine Art wie Phänomene, welche in der im inneren Zeitbewusstsein konstituierten „Ordnungsform der Sukzession“ erscheinen, untereinander in Sinnesfeldern in Beziehung stehen. ${ }^{3}$ Die Assoziation beschreibt damit das Wesen der wechselseitigen Bezogenheit von sukzessiven und gleichzeitigen Erscheinungen. Das zeitliche „Nacheinander“ ist somit die allgemeinste Formierung von Erscheinendem überhaupt, worauf aufbauend die Phänomene nach „Ähnlichkeit“" assoziativ strukturiert werden. So werden die Erscheinungen in der reinen Passivität phänomenologisch als homogen und heterogen ausweisbar. ${ }^{4}$

Diese so hervortreten Erscheinungen sind hier noch bloße „Reize“, welche auf das radikal passive Bewusstsein einwirken. Diese Affektion ist jedoch bereits eine zweiseitige, da sie zwar vom Gegebenen ausgeht, jedoch „das Affiziertsein des Ichs“ impliziert. ${ }^{5}$ Husserl charakterisiert also selbst die pure Passivität in der vorprädikativen Erfahrung bereits als eine im weitesten Sinne subjektive Domäne. Die Welt zeigt sich folglich in ihrer wesenhaften Bezogenheit zum Bewusstsein, dass „,notwendige Bedingung“ ihrer Konstitution ist.

An die Affizierung schließt sich nun die eigentliche Rezeptivität des Ichs an. Diese besteht im Folgeleisten des Ichs, welches sich aktual einem affizierenden Gegenstand zuwenden kann. Dieser Zustand ist näher charakterisierbar als „Wachheit des Ichs“" in Form eines potentiellen oder vollzogenen Folgeleistens des Reizes. ${ }^{6}$ Es ergibt sich eine scheinbare begriffliche Spannung zwischen Affektion/Rezeptivität und Aktualität/Zuwendung. Diese

\footnotetext{
${ }^{1}$ Ebd., 79.

${ }^{2}$ Ebd., 74-75.

${ }^{3}$ Ebd., $77-78$.

${ }^{4}$ EU, 78-79. Die Homogenität und Heterogenität tritt in verschiedenen Intensitäten vor. Grundlegend heterogen sind verschiedene Sinnesfelder wie das Akustische und Optische. Höhergehend unterscheiden sich dann einzelne Erscheinung innerhalb dieser Sinnesfelder. So sind Klang und Optik grundlegender phänomenal verschieden, als beispielsweise zweifarbige Oberflächen, die stets noch ,ineinander übergehen.“

${ }^{5}$ Ebd., 82.

${ }^{6}$ Ebd., 81, 86.
} 
löst sich auf, da die Rezeptivität als „unterste Stufe ichlicher Aktivität“ ausgewiesen wird. ${ }^{1}$

Dieses Interesse „im weiten Sinn“ ist allerdings weiterhin rein affektiv und kein Willensakt, sondern schlicht ein grundlegender Zug der Erfahrung. ${ }^{2}$ Auf es aufbauend entwickeln sich die Ichtendenzen von Aufmerksamkeit und Erfahrungsinteresse. In der Aufmerksamkeit wird der affizierende Gegenstand als Ganzes erstmalig als intentionaler Gegenstand Ziel des ichlichen Fixierung. ${ }^{3}$

Jede Erscheinung lässt sich phänomenologisch mit dem Schema ,impressional (oder präsentational) - retentional - protentional' beschreiben. Das Phänomen ist nicht nur impressional gegeben, sondern verweist in seiner Gegebenheit retentional auf vorhergehende Erscheinungen und protentional auf seine mögliche ichliche Ergründung. So ergibt sich das durch die horizontmäßig mitgegebenen Ergründungsmöglichkeiten begründete „Interesse am Erfahrenen," das Erfahrungsinteresse. ${ }^{4}$ Ist der Gegenstand Ziel der Aufmerksamkeit kann dieser im Erfahrungsinteresse als Gleichbleibender in verschiedenen Abschattungen horizontal und kinästhetisch ergründet werden. ${ }^{5}$

Ist ein Gegenstand intentional als ganze Einheit in der Erfahrung vorkonstituiert und Ziel von Aufmerksamkeit und Erfahrungsinteresse, kann sich eine Modalisierung der Gegebenheitsgewissheit vollziehen. Erfahrung und Ausgangspunkt für jede intentionale Modifikation ist eine ursprüngliche „Seinsgewissheit“. ${ }^{6}$ Dieser Begriff beschreibt die subjektive Setzung „seiend“, welche sich präreflexiv in jeder subjektiven Einsicht in die unmittelbare Gegebenheit eines Gegenstandes mitvollzieht. Auch die Ergründung des Gegenstandes im Erfahrungsinteresse vollzieht sich daher notwendigerweise in Seinsgewissheit. In dem Vollzug des Erfahrungsinteresses ergeben sich hiervon ausgehend nun zwei für das logische Urteilen grundlegende intentionale Modifikationen. Erstens entwickeln sich Erwartungsintentionen, die über unmittelbar zugängige Abschattung selbstgegebener Gegenstände hinaus So-Seiendes erwarten. Diese werden gegebenenfalls im Vollzug des Erfahrungsinteresses enttäuscht — der Gegenstand affiziert anders als erwartet. Hier lässt sich das vorprädikative Fundament der Negation aufweisen. Zweitens entwickeln sich Möglichkeits- und Zweifelsbewusstsein.

\footnotetext{
${ }^{1}$ Ebd., 83.

2 Ebd., 91.

${ }^{3}$ Ebd., 84-85.

${ }^{4}$ Ebd., 96.

${ }^{5}$ Ebd., 86-91.

${ }^{6}$ EU., 23-25.
} 
Hierbei gibt lässt sich die offene Möglichkeit bloßer Unbestimmtheit (beispielsweise eine unbestimmte Röte) von der problematischen Möglichkeit konkurrierender Erwartungsintention unterscheiden (Beispielsweise eine Silhouette, die Mensch oder Puppe sein könnte). ${ }^{1}$

\subsubsection{Objektivierung, Explikation und Relation}

In den Ergebnissen der skizzierten Analysen, welch das passive Affiziertwerden von der einsetzenden ichlichen Rezeptivität getrennt haben, offenbart sich eine entscheidende intentionale Konstitutionsleistung. Diese stellt die unterste Stufe der Objektivierung dar, welche das Fundament für die den „Erkenntnisbesitz“ prädikativer Gegenstandserkenntnis bildet. Denn wo „die Rede von Objekt, einem Gegenstand“ in der puren Passivität noch „eine uneigentliche“" war, ${ }^{2}$ setzen die Analysen der Aufmerksamkeit, des Erfahrungsinteresses und besonders der Modalisierung bereits mehr als bloße Affektion, nämlich eine als solche erkannte gegenständliche Einheit in der Erfahrung voraus.

Am Beispiel der äußeren Wahrnehmung führt Husserl nun darauf aufbauend zwei zentrale Begriffe ein, welche die vorprädikative intentionale Objektivierung phänomenologisch greifbar machen. Erstens die schlichte Erfassung, sowie zweitens die Explikation zum Innen- oder Außenhorizont. Diese stellen neben der Negation und Modalisierung die wichtigsten Momente in der vorprädikativen Ursprungsklärung der Logik dar. ${ }^{3}$

Das schlichte Erfassung beschreibt das niederstufigste unfundierte intentionale Interesse, welches auf den Gegenstand als Ganzes geht. Es setzt also bei der Erfassung eines Gegenstandes als Einheit in den unbestimmt einströmenden Affizierungen an. Schlichtes Erfassen setzt also bereits bei der Aufmerksamkeit und dem Erfahrungsinteresse an. Der intentional objektivierte Gegenstand strukturiert nun die Erfahrung. Das subjektives „Im-GriffBehalten" lässt den Gegenstand über die Retention hinaus erfahrungsstrukturierend weiterwirken, wo er nun ebenfalls ,schlicht betrachtet“" werden kann. ${ }^{4}$

Dies ermöglicht nun die Explikation eines Gegenstandes. Dieser kann intentional im Griff behalten werden und in der betrachtenden Wahrnehmung näher bestimmt werden. Daran anschließend vollzieht sich eine Explikation

\footnotetext{
${ }^{1}$ Ebd., 93-108.

2 Ebd., 81.

${ }^{3}$ EU., 112,

${ }^{4}$ Ebd., 116-121.
} 
hinsichtlich seines „Innenhorizontes“. ${ }^{1}$ Dabei zieht sich der Gegenstand nichtmehr bloß als gleichbleibend durch die Sukzession und Heterogenität der Sinnesfelder, sondern seine intentional erfassten „Momente“ und „Stücke“ zeigen sich als ihm zugehörig. ${ }^{2}$ In dieser ,explikativen Synthesis“ des aktiv im Griff gehaltenen Gegenstandes mit seinen Explikaten ist der Gegenstand Thema der anschließenden Explikationen. In diesem Prozess synthetisiert er sich passiv mit seinen Explikaten, die als seine Bestimmungen in ihn einfließen. ${ }^{3}$ In dieser liegt die vorprädikative Grundlage der Urteilskategorien des Substrates, als Gegenstand der Bestimmungen erhält, sowie der Bestimmung, als einem Substrat synthetisch zugeordnete Eigenschaft. ${ }^{4}$ Der Ablauf der Explikation ist dabei nie willkürlich, sondern verläuft entlang bestimmter "Typiken“. Diese sedimentieren sich im Erfahrungsprozess und fungieren so als „leere Sinnesrahmen" von Erfahrung und Interesse. ${ }^{5}$ „Wahrnehmungsgegenstand“, „Ton einer bestimmten Oktave“, „Ton einer bestimmten Oktave auf einem bestimmten Instrument" können alle Bezeichnung verschieden spezifischer Formen eines sedimentierten Typus dar und evozieren so verschiedene Erwartungen. ${ }^{6}$

Obwohl Substrate zu Bestimmungen und Bestimmungen zu Substraten anderer intentionaler Explikationen werden können, lassen sich auch drei Stufen von absoluten vorprädikativen Substraten bestimmen. Der Begriff des „absoluten Substrates“ umfasst dabei erstens im strengen Sinne den universellen Explikationshorizont (,All-Natur"), welcher selbst nicht als Explikat auftreten kann, zweitens im weiteren Sinne die raumzeitlichen Körper als schlichte Gegenstände der sinnlichen Wahrnehmung, und drittens ,im laxen Sinne" alle prädikativen Konstitutionsleistungen von logischen „Gegenständlichkeiten“, welche in schlicht gegebenen Gegenstände fundiert sind.

\footnotetext{
${ }^{1}$ Ebd., 114-115.

2 Momente eines Gegenstandes existieren nur im Verbund des Gegenstandes, wohingegen Stücke selbst als individuelle Gegenstände Ziel von Intentionen werden können.

${ }^{3}$ Ebd., 128-129. Husserl spricht hier auch von einer Überschiebung von Gegenstand und Explikaten sowie einem „Doppelstrahl der Aufmerksamkeits- und Erfahrung“, der auf den Substratgegenstand als Thema und seine Explikate als Bestimmungen gleichzeitig zielt.

${ }^{4}$ Ebd., 125-127.

${ }^{5}$ Ebd., 141.

${ }^{6}$ Ebd. $65,114,125-126,139-150$.

${ }^{7}$ EU, 159-160. Siehe Kapitel 4 für eine nähere Betrachtung der Analyse logischer Gegenständlichkeiten.
} 
Ein Gegenstand ist durch intentionales Im-Griff-Behalten ferner nicht nur in direkter Nachfolge seiner impressionalen Gegebenheit explizierbar, sondern die Deckungssynthesen der Explikation können ebenfalls in Antizipation, Erinnerung oder im Phantasiebewusstsein vollzogen werden. ${ }^{1}$

Bei der vorprädikativen Bestimmung hinsichtlich gegenständlicher Außenhorizonte, bilden sich „relative Bestimmungen“ von Gegenstanden, welche Husserl von dem rein innenhorizontalen Vorgang der Explikation abgrenzt. ${ }^{2}$ Diese Bestimmungen sind Ergebnisse der untersten urteilenden Aktivitäten über die Beziehungen zwischen Gegenständen, welche wiederum an die assoziative Strukturierung von Phänomenen ansetzt, die bereits in der puren Passivität aufzuweisen ist. Diese Strukturierung vollzieht sich nach Ähnlichkeit und Verschiedenheit in Sinnesfeldern und hat ihrerseits ihre Grundlage in der ursprünglichsten Form der Identitätskonstitution von Erscheinendem, der sukzessiven Differenz und Gleichzeitigkeit im inneren Zeitbewusstseins. Darauf aufbauend werden nun höherstufig Beziehungen zwischen im Griff behaltenen Gegenständen und anderen affizierenden Gegenständen intentional beurteilt. ${ }^{3}$ So sind auf Basis der objektivierenden Intentionen vergleichende Betrachtungen des Im Griff behaltenen Gegenstandes mit weiteren Erfahrungsgegenständen möglich. Gegenstände werden so intentional beispielsweise als "kleiner als“ oder "heller als“ verglichen. Folglich bildet relationale Urteilsaktivität bereits in der vorprädikativen Sphäre der Rezeptivität einen integralen Bestandteil der Welterfahrung.

\subsection{Das prädikative Denken und die logischen Gegenständlichkeiten}

\subsubsection{Das prädikative Urteil}

Das prädikative Denken schließt genetisch an die vorprädikative Sphäre und die in ihr vollzogenen Objektivierungen und Bestimmungen an. ${ }^{4}$ Obwohl vorprädikative Erfahrung und Prädikation "faktisch in der Regel eng ineinander verflochten sind", 5 sind sie dennoch analytisch scharf voneinander zu trennen. Im prädikativen Denken zeigt sich höhere Stufen ichlicher objektivierender und bestimmender Aktivität. Die Prädikation schaffen

\footnotetext{
${ }^{1}$ Ebd., 143-145.

2 Ebd., 115.

${ }^{3}$ Ebd., 179-187, 224-227.

${ }^{4}$ EU, 239-241.

${ }^{5}$ Ebd., 239.
} 
erstmals von der Rezeptivität losgelösten, bleibenden Erkenntnisbesitz, welcher das entstandene Erkenntnisinteresse erfüllt. ${ }^{1}$ Wie Husserl explizit darlegt, darf eine phänomenologische Analyse der Prädikation und ihrer neuen epistemischen Leistung nicht erst an sprachlichen Urteilen ansetzen, da diese ihrerseits in grundlegenderen prädikativen Konstitutionsleistungen fundiert sind. ${ }^{2}$

Die Prädikation beginnt mit dem Erkenntnisinteresse, welches im Gegensatz zu den vorprädikativen Interessen nicht bloß rezeptiv einen vorgegebenen Gegenstand als solchen betrachtet oder horizontal expliziert, sondern willentlich bleibenden Erkenntnisbesitz von ihm anstrebt. Nachdem bereits vorprädikativ in der Explikation Gegenstände konstituiert und synthetisch verknüpft wurden, richtet sich das Erkenntnisinteresse nun „mehrstrahlig “ auf den Substratgegenstand und seine Bestimmungen zurück, wie sie als Ergebnis dieser Synthese als Substrat und Bestimmungen verbunden sind. ${ }^{3}$ Durch diese aktive Nachverfolgung der passiven vorprädikativen Synthese wird das Erkenntnisinteresse befriedigt, da sich nun der Erkenntnisgewinn der Bestimmung zeigt. Dieser wird im prädikativen Urteil ,S ist p ' festgehalten. ${ }^{4}$ Bereits prädikativ beurteile Gegenstände können erneut expliziert und prädikativ bestimmt werden können. Dabei bleibt jedoch die Form des prädikativen Urteils immer als epistemische „Urzelle“,$S$ ist $p^{\text {“ }}$ identisch. ${ }^{5}$ Die Prädikation ist wesenhaft „Zweigliedrig“ auf ein Substrat und seine Bestimmungen bezogen. ${ }^{6}$ In der vollzogenen prädikativen Erfassung zeigt sich eine wesenhafte Differenz zwischen der Selbstständigkeit des Substrates (Substantivität) und der Unselbstständigkeit der Bestimmung (Adjektivität) $^{7}$ Auch prädikative Beziehungsurteile stellen hier keine Ausnahme dar, obwohl sie die Prädikationen der Beziehung von zwei selbstständig erfassten Gegenständen sind. Es ist dabei stets ein Selbstständiges als Substrat $\left(S^{\circ}\right)$ erfasst und seine Beziehung $\mathrm{zu}$ dem anderen Gegenstand wird als prädikative Bestimmung (,ist $p^{\circ}$ ) adjektiviert. ${ }^{8}$

\footnotetext{
${ }^{1}$ Ebd., 231-234.

2 Ebd., 247.

${ }^{3}$ Ebd., 245.

${ }^{4}$ Ebd., 242-246.

${ }^{5}$ Ebd., 250.

${ }^{6}$ Ebd., 242.

7 Ebd., 247-249. Diese kann in anschließenden, die unselbstständige Bestimmung substantivierenden Hat-Urteilen zu teilen relativiert werden.

${ }^{8}$ EU., 266-277.
} 
Die aktive prädikative Bestimmung eines Substrates ist zwar in der Rezeptivität verankert, aber dennoch eine „spontane“ Leistung. Ein Identisches Substrat , $S^{6}$ zeigt als Resultat einer subjektiven Modifikation nicht mehr in einer bestimmten prädikativ festgehaltenen Sachlage als $S_{q}, S_{p}$ oder $\mathrm{S}_{\mathrm{aq}}$, sondern in einer die verschiedenen prädikativen Bestimmungen durchziehenden Funktion als gleichbleibendes Urteilsubjekt mit verschiedenen möglichen Bestimmungen. Die genetische Besonderheit des bleibenden Erkenntnisbesitzes des prädikativen Urteils lässt sich durch die Entwicklung eines „logischen Sinns“ des Gegenstandes beschreiben. Dieser geht in seinem epistemischen Gehalt über die vorprädikativen Deckungssynthesen hinaus, da der Gegenstand nun nicht mehr bloß als Einheit der Wahrnehmung, sondern als verschiedene feste Bestimmungen enthaltend begriffen ist. Über die typische Vorbekanntheit, sein Im-Griff-behalten und seinen Explikationsvorgang hinaus, kann der Gegenstand $\mathrm{S}$ nun auch durch spontane subjektive Leistungen als $\mathrm{S}_{\mathrm{q}}, \mathrm{S}_{\mathrm{p}}$ oder $\mathrm{S}_{\mathrm{aq}}$, antizipiert oder erinnert werden. ${ }^{1}$

\subsubsection{Logische Gegenständlichkeiten}

In der spontanen subjektiven Leistung der Prädikation vollzieht sich nicht nur die Erfassung von Gegenständen als Selbstständige mit unselbstständigen Bestimmungen. Durch das prädikative Urteil, welche dieses Verhältnis als ,S ist $p^{\prime}$ festhält, konstituiert sich außerdem ein neuer Gegenstandstypus vor, die logischen Gegenstände, welche Husserl zur Hervorhebung ihrer ontologischen Besonderheit als „logische Gegenständlichkeiten“ beschreibt. ${ }^{2}$ Deren spontane Konstitution vollzieht durch eine Substantivierung der ursprünglich mehrstrahligen intentionalen Einstellung, $\mathrm{S}+\mathrm{p}_{1}+\mathrm{p}_{2}+\ldots+\mathrm{p}_{\mathrm{n}}{ }^{6}$ in der Prädikation. ${ }^{3}$ Die im prädikativen Urteil aktuell erfasste Sachlage, „,das Geurteilte“, ist nun intentional einstrahlig als neuer intentionaler Gegenstand konstituiert, als Sachverhalt: „Sachverhalte sind die Korrelate von Urteilen, das heißt sie konstituieren sich ursprünglich nur in Urteilen. “4 Der Sachverhalt ist also als logischer Gegenständlichkeit wesenhaft korrelativ zum noetischen Urteil und besitzt ein unabhängiges gegenständliches Sein. Als solcher kann er auch selbst Substrat neuer Urteile werden. ${ }^{5}$ Es ist der Sachverhalt, welcher als

\footnotetext{
${ }^{1}$ Ebd., 276-280.

2 Der Einfachheit halber wird im Folgenden immer von logischen Gegenständlichkeiten die Rede sein, obwohl Husserl auch und zumeist von kategorialen Gegenständlichkeiten oder Verstandesgegenständlichkeiten spricht.

${ }^{3}$ Ebd., 282-288.

${ }^{4}$ Ebd., 289.

${ }^{5}$ EU., 296-299.
} 
bleibender Erkenntnisbesitz potentiell sprachlich kommunizierbar ist und der in den Sätzen der Logik sprachlich verhandelt wird. ${ }^{1}$

Die in Sachverhalten substantivierten logischen Relationen ${ }^{2}$ geben die „geurteilten“ Sachlagen von Gegenständen und Bestimmungen wieder. Da sie genetisch auf diesen aufbauen, werden sie von Husserls auch als „Sinngegenständlichkeiten" bezeichnet, deren Sinn immer über die Prädikation zurück zur Gegebenheit in der Erfahrung erweist. ${ }^{3}$ Die ständig in der erkennenden und handelnden Praxis modalisierten und außer Kraft gesetzten (negierten) prädikativen Urteile, verweisen daher in ihrer letzten Evidenz ebenfalls auf die Selbstgegebenheit ihrer Substrate und Bestimmungen in der Wahrnehmung: „In keinem Erfahrungsurteil, und mag es noch so sehr Erfahrungssattheit haben, kann das Urteilen das Wahre, den Sachverhalt selbst, in sich tragen. “4

Die logischen Gegenstände weisen im Unterschied zu realen Gegenständen eine „Verschiedenheit der Zeitlichkeit“ auf. ${ }^{5}$ Auch sie konstituieren sich notwendigerweise in der inneren Zeitlichkeit des Bewusstseinsflusses. Solange die Gegenstände eines Sachverhalts selbst keine logischen Gegenstände sind, ist außerdem ihr Sinn ebenfalls auf die objektiv-messbare Zeit der Wahrnehmungsgegenstände gerichtet. Schließlich werden sie aus prädikativen Urteilen über Erfahrungsgegenstände erst ,entnommen“. 6 Entgegen der Ontologie realer Gegenstände, ist es logischen Gegenständen eine Individuation in der objektiven Zeit nicht wesenhaft. Sie nicht in bestimmten intersubjektiv einsehbaren Zeitdauern gegeben. Der Sachverhalt ,Die Wiese X ist grün' weist nicht den objektiv-zeitlichen Moment auf, in

\footnotetext{
${ }^{1}$ Für Husserl verlangt sprachlichen Kommunikation jedoch zusätzliche bedeutungsgebende Akte, welche prädikative Erkenntnis auf Basis sprachlicher Zeichen wirklich kommunizierbar macht. Die Bedeutung von Zeichen ist der irreale Sachverhalt. Für Husserls Theorie der Bedeutung bzw. bedeutungsgebender Akte siehe Dietmar Lohmar, Denken ohne Sprache: Phänomenologie des nicht-sprachlichen Denkens bei Mensch und Tier im Licht der Evolutionsforschung, Primatologie und Neurologie (Basel: Springer, 2016), 33-37; Robert Sokolowski, Husserlian Meditations: How Words present Things (Evanston: North Western University Press, 1974), 115.

${ }^{2}$ Der Begriff der Relation umfasst hier nicht bloß die bereits erläuterten Beziehungsurteile, sondern in einem weiten Sinn alles prädikativ aufeinander Bezogene. Darin sind Beziehungsurteile im strengen sind genauso enthalten wie kopulative Bestimmungen (etwa das grundlegende,$S$ ist $\mathrm{p}^{\circ}$ ).

${ }^{3}$ Ebd., 323.

${ }^{4}$ Ebd., 347.

${ }^{5}$ Ebd., 303.

${ }^{6}$ Ebd., 282.
} 
welchem ich die Grüne der Wiese $\mathrm{X}$ beurteile, beziehungsweise die Wiese $\mathrm{X}$ wirklich grün ist. Er kann auch Jahre später und unabhängig von seiner Geltung intentional gegeben sein und in seiner Gewissheit affirmiert, in Frage gestellt oder ausgeschaltet werden. Beurteilt wird zwar die Sachlage der in der objektiven Zeit wahrgenommenen Wiese X, aber das substantivierte Geurteilte ist folglich als Sachverhalt ontologisch nicht in der objektiven Zeit realer Gegenstände verortbar - Der Sachverhalt ist ein irrealer Gegenstand. ${ }^{1}$

Die formale Logik hat mit ihren Sätzen also Sachverhalte und damit die Korrelate prädikativer Urteile als Objekte, deren präsumtive und apodiktische Gewissheitsbedingungen sie untersucht. ${ }^{2}$ Diese sind nicht ursprünglich oder notwendig sprachlich gegeben und konstituieren sich in den subjektiven Aktivitäten des Urteilens und Substantivierens. Diese haben wiederum ihren Ursprung in der vorprädikativen noetischen Erfassung eines Gegenstands sowie in den vorprädikativen passiven Deckungssynthesen von Gegenständen mit ihren assoziativen Verweisungen und Explikationen. Nicht nur der Sinn logischer Gegenstände verweist zurück in die vorprädikative Erfahrung, denn auch Gewissheit, Infragestellung und Negation von prädikativen Urteilen sind ohne die ursprüngliche Modalisierung des passiven Seinsglaubens und die Enttäuschung von Erwartungsintention nicht möglich.

\section{Resümee: Vom Schweigen zur Ontologie}

Wie sich gezeigt hat, führt Wittgenstein Klärungsversuch der Sprache ihn letztendlich auf die allgemeine logische Form als Bedingung der Möglichkeit jeder Erkenntnis. Die philosophisch zu beseitigenden unsinnigen Sätze ergeben sich, wenn die intersubjektiven Verwirrungen der Alltagssprache Zeichen aus ihrem bezeichnenden Satzzusammenhang loslösen und sie auf unsinnige Weise propositional verknüpfen. Alle anderen Sätze bilden die Welt durch ihre empirische Falsifikation oder Verifikation ab. Die Möglichkeit der Beschreibung der Welt setzt jedoch einen Umstand voraus, der selbst nicht als zu bezeichnender Referent in der Welt vorhanden ist. Die logische Form, die Satz und Sachlage verbindet und Bezeichnung möglich macht. Diese zeigt sich

\footnotetext{
${ }^{1}$ EU., 309.

2 Präsumtive Gewissheit bezeichnet die Offenheit für Gewissheitsmodalisierung. Apodiktische Gewissheit ist lediglich die Gewissheit der eidetischen Einsicht in das Wesen von Gegenständen. Logische Schlüsse sind apodiktisch gewiss. Husserls Analyse des Allgemein-Urteilens, zu welchen die Wesenschau abschließend zählt, wird hier nicht behandelt.
} 
in Denken und sinnvoller Sprache. Würde es diese transzendentale logische Form nicht geben, wäre jedweder Sinn ausgeschlossen. Wittgenstein positioniert seinen Logiktranszendentalismus folglich als Gegenpol zum Postulat einer außerlogischen und somit außerepistemischen, unzugänglichen Wirklichkeit. Jede so argumentierende Theorie wäre nicht nur kontraintuitiv, sondern schlicht nicht sinnvoll formulierbar, da ihre Geltung bereits Verifizierbarkeit und dies wiederum eine logisch zugängliche Sachlage voraussetzt.

Dies parallelisiert einem Hauptanliegen Husserls, welcher die Infragestellung von einer subjektiv zugänglichen Welt der Erscheinungen als „vollkommenen Widersinn“ bezeichnet. ${ }^{1}$ Im Gegensatz zum Tractatus weist Erfahrung und Urteil die Logik jedoch als sprachlich analysierbar und keineswegs transzendental aus. Logische Gegenständlichkeiten sind nicht Bedingung der Möglichkeit von Erkenntnis, sondern selbst vorlogisch bedingter Erkenntnisbesitz. Husserls Weg vorbei an der epistemischen Unzulänglichkeit ist folglich ein grundlegend verschiedener.

Dies lässt sich zurückführen auf Husserls genetische, konstitutive Phänomenologie und die daran gebundene Unterscheidung zwischen Arten der Evidenz. Von der „korrelationalistischen“2 Prämisse einer auf die Welt bezogenen transzendentalen Subjektivität, lassen sich so verschiedene Formen der Evidenz realer Gegenstände und irrealer Sachverhalte unterscheiden. Die Gewissheit und Modalisierung realer Gegenstände unterscheidet sich von der Gewissheit irrealer Sachverhalte, die als Aktkorrelate bleibenden Erkenntnisbesitz schaffen. Erst letztere lassen sich als Repräsentationen von Sachlagen verstehen, die folglich im allgemeinen Verständnis der formalen Logik negiert und affirmiert werden können, da sie als eigener intentionaler Gegenstand Stellungnahmen hervorbringen können. Erstere hingegen sind ontologisch nicht durch eine Bildrelation (im weitesten Sinne) von den Sachlagen getrennt, sondern bestehen in Einstellungsänderungen gegenüber den intentionalen Gegenständen selbst. Die klassische Negation und Affirmation der formalen Logik setzt für Husserl also eine substantivierte irreale Sachlage worauf, zu der eigenständig in einem „Wahrheitsurteil“" Stellung bezogen werden kann. ${ }^{3}$

${ }^{1}$ Edmund Husserl, Zur phänomenologischen Reduktion: Texte aus dem Nachlass (1926-1935) (Dordrecht: Kluwer Academic, 2002), 402.

2 Maximilian Beck, „Die neue Problemlage der Erkenntnistheorie,“ Deutsche Vierteljahrsschrift für Literaturwissenschaft und Geistesgeschichte 6,4 (1928), 611639. Zahavi, Husserl's Legacy, 114. Dieser ursprünglich von Maximilian Beck vorgeschlagenen Begriff bezeichnet die grundlegende phänomenologische Interdependenz von Subjekt und Welt.

${ }^{3} \mathrm{EU}, 354-358$. 
Im Tractatus ist die Gewissheit und Modalisierung der Logik in einem allumfassend binären Schema wahr-falsch allerdings bereits konstitutiv für jeden möglichen epistemischen Akt, da ein solcher immer Sachlagen richtig oder falsch abbildet („Man kann keinen Unsinn urteilen“l). Obwohl das Denken unmittelbar sinnvoll bei den Sachlagen ansetzte und die Grenzen der Welt bestimme, bleibt es dabei jedoch von der Welt durch eine Bildrelation getrennt. Wittgenstein projiziert also intentionale Modalisierungen der höherstufigen Gewissheit von Sachverhalten auf die bereits vorprädikativ erfassbaren realen Gegenstände. Dadurch, dass er unmittelbare Erfahrung im Denken bereits als Repräsentation versteht, konzipiert er eine BildAbgebildetes Differenz, welche nur durch einen Logiktranszendentalismus überwinden werden kann, welcher die epistemische Zugänglichkeit der Welt rettet. Wittgenstein bewahrt seinen Korrelationalismus vor der Aporie eines kantischen Ding-an-sich, jedoch nur auf Kosten einer Reduktion von aller Evidenz auf syntaktische Verifikation und Falsifikation. So erscheint alle Gewissheit als Bestanteil einer mystischen logischen Form, welche alle genetischen Gewissheitsdifferenzen nivelliert und dabei ontologisch unbestimmbar bleibt.

Husserls entscheidende Feststellung liegt also darin, dass die die Evidenz prädikativer logischer Urteile phänomenologisch in der Gewissheit realer Gegenstände, abschließend im passiven Seinsglauben begründet liegt, jedoch trotzdem scharf zwischen den jeweiligen noematischen Gegebenheitsweisen und noetischen Modalisierungen in vorprädikativer Erfahrung und prädikativem Denken unterschieden werden muss. So ist nicht nur die Konstitution der Gegenstände der Logik ontologisch analysierbar. Vielmehr wird deren ganze epistemische Besonderheit, die Schaffung eines bleibenden, kommunizierbaren Erkenntnisbesitzes erst im Kontrast zur vorprädikativen Erfahrung deutlich.

\section{Literatur}

Ayer, A.J., „The Elementary Propositions of the Tractatus,“ in Sprache und Ontologie: Akten des 6. Internationalen Wittgenstein Symposiums 23. bis 30. August 1981, Kirchberg/Wechsel, Österreich, hsrg. v. Elisabeth Leinfellner et. Al. (Wien: Hölder-Pichler-Tempsky, 1992), 419-428.

Beck, Maximilian, „Die neue Problemlage der Erkenntnistheorie,“ Deutsche Vierteljahrsschrift für Literaturwissenschaft und Geistesgeschichte 6,4 (1928), 611-639.

${ }^{1}$ TLP, $§ 5.5422$. 
Brudzinska, Jagna, „Erfahrung und Urteil,“ In Husserl Handbuch: Leben, Werk, Wirkung, hrsg. v. Sebastian Luft u. Maren Wehrle (Stuttgart: J.B. Metzler, 2017), 104-113.

Conant, James u. Bronzo, Silver, „Resolute Readings of the Tractatus,“ In A Companion to Wittgenstein hrsg. v. Hans-Johann Glock u. John Hyman (WestSussex: Wiley-Blackwell, 2017), 175-195.

Doyon, Maxime, „Philosophy of Mind,“ In Husserl Handbuch: Leben, Werk, Wirkung, hrsg. v. Sebastian Luft u. Maren Wehrle (Stuttgart: J.B. Metzler, 2017), 320-325.

Drummond, John J., „Intentionality without Representationalism,“ In The Oxford Handbook of Contemporary Phenomenology, hrsg. v. Dan Zahavi (Oxford: Oxford UP, 2012), 115-133.

Frege, Gottlob Begriffschrift (Halle: Louis Nebert, 1879).

Gabriel, Markus, Warum es die Welt nicht gibt (Berlin: Ullstein, 2013).

Habermas, Jürgen, Zur Logik der Sozialwissenschaften (Frankfurt a.M.: Suhrkamp, 1982).

Husserl, Edmund, Die Krisis der europäischen Wissenschaften und die transzendentale Phänomenologie: Eine Einleitung in die phänomenologische Philosophie (Hamburg: Meiner, 2012).

Husserl, Edmund, Erfahrung und Urteil: Untersuchungen zur Genealogie der Logik (Hamburg: Felix Meiner, 1972).

Husserl, Edmund, Erste Philosophie I (Dordrecht: Kluwer, 1996).

Husserl, Edmund, Texte zur Phänomenologie der Intersubjektivität II (Den Haag: Nijhoff, 1973).

Husserl, Edmund, Zur phänomenologischen Reduktion: Texte aus dem Nachlass (1926-1935) (Dordrecht: Kluwer Academic, 2002).

Kremer, Michael, „Russell’s Merit,“'In Wittgenstein’s early Philosophy, hrsg. v. José Zalabardo (Oxford: Oxford UP, 2012), 195-240.

Kremer, Michael, „The Whole Meaning of a Book of Nonsense: Reading Wittgenstein's Tractatus," In The Oxford Handbook of the History of Analytic Philosophy, hrsg. v. Michael Beaney (Oxford: Oxford UP, 2013), 451-487.

Lohmar, Dieter, Denken ohne Sprache: Phänomenologie des nicht-sprachlichen Denkens bei Mensch und Tier im Licht der Evolutionsforschung, Primatologie und Neurologie (Basel: Springer, 2016).

Lohmar, Dieter, Erfahrung und kategoriales Denken: Hume, Kant und Husserl über vorprädikative Erfahrung und prädikative Erkenntnis (Dordrecht: Springer, 1999).

Lohmar, Dieter, „Zu der Entstehung und den Ausgangsmaterialien von Edmund Husserls Werk Erfahrung und Urteil,“ Husserl Studies 13,1 (1996), 31-71.

Lohmar, Dieter, „Warum braucht die Logik einer Theorie der Erfahrung?“" In Phenomenology on Kant, German Idealism, Hermeneutics and Logic: Philosophical Essays in Honor of Thomas M. Seebohm (Dordrecht: Springer, 2000), 149-170.

Mohanty, J.N., Edmund Husserl's Theory of Meaning (Dordrecht: Martinus Nijhoff, 1964). 
Robinson, Howard, „Coda B: Analytic versus Continental,“ In The Bloomsbury Companion to Analytic Philosophy, hrsg. v. Ders. u. Barry Dainton (London: Bloomsbury, 2013).

Russell, Bertrand u. Whitehead, Alfred North, Principia Mathematica, Bd. 1-3 (Cambridge: Cambridge UP, 1927).

Russell, Bertrand, „On Denoting,“ Mind 14,56 (1905), 479-493.

Sokolowski, Robert, Husserlian Meditations: How Words present Things (Evanston: North Western University Press, 1974).

Weismann, Friedrich, McGuiness, Brian F. (Hrsg.), Ludwig Wittgenstein und der Wiener Kreis: Gespräche, aufgezeichnet von Friedrich Waismann (Frankfurt a.M.: Suhrkamp, 1984).

Woodruff Smith, David u. Thomasson, Amie L. (Hrsg.), Phenomenology and Philosophy of Mind (Oxford, Oxford UP, 2005).

Zahavi, Dan, Husserl's Legacy: Phenomenology, Metaphysics, and Transcendental Philosophy (Oxford: Oxford UP, 2017).

Zahavi, Dan, „Internalism, Externalism, and Transcendental Idealism,“ Synthese 160 (2008), 355-374. 